\title{
Sinergismo en la respuesta inmune en ratones BALB/c//BIOULA con parasitosis combinadas: Syphacia obvelata y Leishmania mexicana
}

Rosa De Jesús*, Lic. ${ }_{1}$, Willmer Pavón, Lic. ${ }_{1}$

${ }_{1}$ Bioterio, Universidad de Los Andes, Mérida, Venezuela

Recibido: 11 de octubre del 2014. Aprobado: 15 de diciembre del 2014.

*Autor de correspondencia: Rosa De Jesús. Bioterio, Universidad de Los Andes, Mérida, Venezuela. Apartado postal 5101, teléfono: 582742403128. Correo electrónico: rosadej@ula.ve

Cómo citar este artículo: De Jesús R, Pavón W. Sinergismo en la respuesta inmune en ratones BALB/C//BIOULA con parasitosis combinadas: Syphacia obvelata y Leishmania mexicana. Spei Domus. 2014;10(21):29-39. doi: http://dx.doi.org/10.16925/sp.v10i21.916

Resumen. Introducción: los ratones BALB/c//BIOULA son usados en experimentos en los que se estudian parásitos de las especies Lehismania mexicana, determinando concentraciones de IgG. En estudios previos, se determinó que los ratones parasitados con Syphacia obvelata presentan altas titulaciones de IgG. El objetivo del trabajo fue determinar si existe sinergismo en la respuesta inmunológica de ratones BALB/C//BIOULA con parasitosis combinada. Materiales y métodos: los animales se distribuyeron como Grupo A, sin parásitos; Grupo B, parasitados con Syphacia obvelata; Grupo C, con Leishmania mexicana y Grupo D, con parasitosis combinada. Durante dos meses se realizaron curvas de crecimiento, diagnóstico coproparasitológico, valoración hematológica y titulación de anticuerpos mediante ELISA. Resultados: en las curvas de crecimiento, se observó que los ratones con parasitosis combinadas presentaron la menor ganancia de peso y de talla, y la mayor cantidad de huevos y parásitos adultos de Syphacia obvelata; en los parámetros hematológicos, presentaron diferencias estadísticamente significativas en los valores hallados, y al valorar la concentración de IgG, se observó que la mayor cantidad de unidades de IgG se presentaron igualmente para los sueros de los ratones con parasitosis combinadas. Conclusiones: los resultados de las curvas de crecimiento, las pruebas hematológicas y la titulación de IgG permitieron concluir que existe sinergismo en la respuesta de los ratones al encontrarse parasitados con Syphacia obvelata y Leishmania mexicana. Esto puede conducir a resultados falsos positivos cuando el investigador experimente con Lehismania mexicana y los ratones se encuentren parasitados con Syphacia obvelata.

Palabras clave: animales de laboratorio, bioterio, parasitosis, sistema inmunológico.

Synergism in the Immune Response of BALB/c and BIOUla Mice with Combined Parasitism: Syphacia obvelata and Mexican Leishmania

Abstract. Introduction: BALB/C//BIOULA mice are used in experiments studying parasites of the Mexican Leishmania species, determining IgG concentrations. Previous studies have shown that mice parasitized with Syphacia obvelata present high titers of IgG. The aim of this study was to determine if there is synergism in the immunological response of ВАLВ/C//BIOULA mice with combined parasitism. Materials and methods: The animals were divided into four groups: Group A, without parasites; Group B, parasitized with Syphacia obvelata; Group C, with Mexican Leishmania; and Group D, with combined parasitism. Over two months, the mice were monitored using growth curves, coproparasitological diagnosis, and hematological and antibody titer evaluation through ELISA. Results: The growth curves indicated that mice with combined parasitism gained less weight and size, and possessed the highest quantity of Syphacia obvelata eggs and adult parasites. Statistically significant differences were found in the values of hematological parameters, and measurement of IgG concentration also showed that the highest quantity of IgG units occurred for sera of mice with combined parasitism. Conclusions: the results of the growth curves, the hematological tests and the IgG titers confirmed that there is a synergism in the response of rats parasitized with Syphacia obvelata and Mexican Leishmania. This may lead to false positive results when researchers conduct experiments with Mexican Leishmania on mice that are parasitized with Syphacia obvelata.

Keywords: laboratory animals, vivarium, parasitism, immunological system

\section{Sinergismo na resposta imune em ratos $\mathrm{BALB} / \mathrm{c} / /$ BIOULA com parasitoses combinadas: Syphacia obvelata e Leishmania mexicana}

Resumo. Introdução: os ratos BALB/c//BIOULA são usados em experimentos em que se estudam parasitas das espécies Leishmania mexica$n a$, determinando concentrações de IgG. Em estudos prévios determinou-se que os ratos parasitados com Syphacia obvelata apresentam altas titulações de IgG. O objetivo do trabalho foi determinar se existe sinergismo na resposta imunológica de ratos BALB/c//BIOULA com parasitose combinada. Materiais e métodos: os animais distribuem-se como Grupo A, sem parasitas; Grupo B, parasitados com Syphacia obvelata; Grupo C, com Leishmania mexicana e Grupo D, com parasitose combinada. Durante dois meses realizaram-se curvas de crescimento, diagnóstico coproparasitológico, valorização hematológica e titulação de anticorpos mediante elisa. Resultados: nas curvas de crescimento observou-se que os ratos com parasitoses combinadas apresentaram 
o menor ganho de peso e de tamanho; a maior quantidade de ovos e parasitas adultos de Syphacia obvelata; nos parâmetros hematológicos, apresentaram diferenças estatisticamente significativas nos valores encontrados, e ao valorizar a concentração de IgG, observou-se que a maior quantidade de unidades de IgG apresentou-se igualmente para os soros dos ratos com parasitoses combinadas. Conclusões: os resultados das curvas de crescimento, as provas hematológicas e a titulação de
IgG permitiram concluir que existe sinergismo na resposta dos ratos ao encontrarem-se parasitados com Syphacia obvelata e Leishmania mexicana. Isto pode conduzir a resultados falsos positivos quando o pesquisador experimente com Leishmania mexicana e os ratos encontram-se parasitados com Syphacia obvelata.

Palavras-chave: animais de laboratório, biotério, parasitoses, sistema imunológico.

\section{Introducción}

El poliparasitismo es el fenómeno causado por parásitos pertenecientes a distintas especies, que afectan al hospedador en determinado momento; estos pueden ubicarse o no, en un mismo órgano [1]. El poliparasitismo experimental permite estudiar el sinergismo que se puede desarrollar en un organismo por la acción combinada de su respuesta inmunológica hacia cada microorganismo. Babu et al. [2] demostraron que la presencia de nematodos disminuye la producción de IL-17 e IL-23 involucradas en las respuestas de tipo Th1 para contrarrestar los antígenos de Mycobacterium, beneficiando la infección de lepra tuberculoide. Pereira et al [3] informaron que la presencia de helmintos en pacientes con lepra lepromatosa aumenta el riesgo de infección, debido a que la presencia de Mycobacterium leprae y helmintos desarrolla una respuesta inmunológica de tipo Th2, lo que facilita la infección de ambos.

Estos ejemplos implican evaluar este fenómeno en animales de laboratorio, los cuales son usados en investigaciones en las que se les infecta con parásitos $\mathrm{u}$ otros microorganismos con la finalidad de valorar posibles semejanzas con la etiología en humanos, al evaluar el proceso de la enfermedad y posibles tratamientos, y sin embargo, cuando estos son producidos y mantenidos en condiciones convencionales pueden ser portadores de parásitos comunes a ellos, dando paso al fenómeno de poliparasitismo.

En los últimos años, se han analizado las respuestas humorales y celulares en diferentes cepas de ratones, las cuales difieren en la resistencia/susceptibilidad frente a la infección experimental producida por Leishmania, Stanley [4], en cepas de ratones BALB/c y C57BL/6. En el caso de los ratones BALB/c —estos son altamente susceptibles a L. major y L. mexicana-, la infección produce lesiones nodulares que visceralizan, con producción de anticuerpos, pero sin el desarrollo de una hipersensibilidad tardía [5]. Por el contrario, los ratones $\mathrm{C} 57 \mathrm{BL} / 6$ son resistentes a la infección por L. major o L. mexicana y desarrollan lesiones pequeñas que se curan entre 10 y 12 semanas; son resistentes a la reinfección y desarrollan respuestas inmunes humoral y de hipersensibilidad tardía [6]. De acuerdo con Preston y Dumonde [7], la mayoría de las otras cepas de ratones usados como modelos experimentales son intermedias en la susceptibilidad a este parásito.

En las infecciones causadas por microorganismos, incluimos las infecciones parasitarias intestinales, las cuales son muy comunes en ratones que se crían en bioterios convencionales, entre las que se encuentran más frecuentemente la parasitosis por Syphacia obvelata $[8,9]$; y con respecto a las parasitosis tanto de protozoos, helmintos y ectoparásitos que ocurren en los bioterios, estas presentan una gran morbilidad y en ocasiones mortalidad [10], por lo que es importante el conocimiento de la influencia de las parasitosis en la respuesta inmune frente a estos [11]. En el caso de las infecciones causadas por parásitos intestinales, se ha demostrado en las parasitosis con Syphacia obvelata, que estos producen IgG [12], que el ratón BALB/C//BIOU manifiesta una mayor susceptibilidad por la presencia del parásito y que los ratones de la cepa $\mathrm{C} 57 \mathrm{BL} / 6 / / \mathrm{BIOU}$ presentan resistencia [13].

Contar con animales con calidad microbiológica definida en las investigaciones biomédicas conlleva resultados reproducibles y confiables en diferentes investigaciones; sin embargo, realizar exploración con animales que no cuentan con una microflora conocida y que a la vez esta pueda influir en la experimentación, porque afectan su respuesta inmunológica, puede llevar a falsos resultados [12].

Ciertos parámetros como fisiológicos [14], zootécnicos y hematológicos [15], y concentración de anticuerpos $[12,16,17]$, se pueden emplear como indicadores de los procesos de poliparasitismo.

El objetivo de este trabajo fue determinar la posible sinergia en ratones BALB/c//BIOULA, a la infección de parasitosis combinada: Syphacia obvelata y Leishmania mexicana. 


\section{Materiales y métodos}

Se utilizaron 16 hembras gestantes de ratones BALB/c// BIOULA, y los grupos formados fueron de 4 hembras gestadas por grupo, lo que conllevaría contar con una población de aproximadamente 32 animales una vez nacidas las crías, de las cuales se extraería una muestra de $n=10$ hembras y $n=10$ machos, siendo la población total evaluada de $n=20$. Para esto se consideró la fórmula $\mathrm{N}=\mathrm{Z}_{2} \mathrm{PQ} / \mathrm{E}_{2}$, donde $\mathrm{N}$ es el tamaño de la muestra por usar, $z$ es el nivel de confianza de $95 \%$, P es la probabilidad de encontrar la variable (sinergismo) presente en la muestra, Q es la probabilidad de no encontrar sinergismo en la muestra, y E, el error de muestreo que se está dispuesto a aceptar en el estudio (5\%). El cálculo se realiza considerando la variable que se quiere estudiar que, en este caso, es el sinergismo; para disminuir el error del muestreo, se puede aumentar el número de la muestra para evaluar, por lo que en este caso se llevó el $\mathrm{N}$ final a usar a $n=10$, además de que se eligió por muestreo aleatorio simple.

Las hembras fueron alojadas en grupos de cuatro, cuando se encontraban a término (19 días de gestación), en un "aislador" que mantiene una presión positiva 13 mm de $\mathrm{H}_{2} \mathrm{O}$, previamente esterilizado, una temperatura de $21{ }^{\circ} \mathrm{C}$ y una humedad relativa de $66 \%$. Este aislador se conserva en un macroambiente que proporciona 12 horas luz: 12 horas oscuridad. El alimento suministrado es tratado en autoclave con $1 \mathrm{~min} / 120^{\circ} \mathrm{C} / 21$ atmósferas de presión; la cama es autoclaveada a $10 \mathrm{~min} / 120^{\circ} \mathrm{C} / 21$ atmósfera de presión. Cada grupo de cuatro se trabajó por separado, el aislador se limpiaba y desinfectaba previo al alojamiento de cada grupo.

Luego del parto, se dejaron desarrollar las crías $\mathrm{y}$ tras el destete, se seleccionaron 10 ratones hembras y 10 ratones machos por grupo experimental: Grupo A: control sin la presencia de parásitos; Grupo B: inoculados con Syphacia obvelata; Grupo C: inoculados con Leishmania mexicana y Grupo D: inoculados con Syphacia obvelata y Leishmania mexicana.

Para la realización del ensayo experimental, se usó un total de 66 ratones: 16 hembras grávidas, de las cuales se obtuvieron 40 machos y 40 hembras (grupos experimentales), todos con aproximadamente 2 meses de nacidos. Se usaron, además, 10 ratones machos BALb/c//Bioula de un mes de edad, los cuales se alojaron en el área de experimentación del bioterio, y se mantuvieron sin esterilizar el encamado, el alimento y el agua, y el cambio de cama se realizó una vez por semana. De este grupo se obtuvieron los parásitos Syphacia obvelata, y se usó para la obtención de los parásitos de Lehismania mexicana un hámster BIOu dorado.

A los animales del Grupo control (sin parásitos), primer grupo alojado en el aislador, a partir de la semana de nacidos se les pesó y midió semanalmente, y se les tomó muestra vía retroorbital $(200 \mu \mathrm{L})$, con la finalidad de realizar un conteo de glóbulos blancos, hematocrito y recuento diferencial.

A los animales del Grupo в (inoculados con Syphacia obvelata) se les realizaron semanalmente los exámenes coproparasitológicos a partir de los 15 días de nacidos, mediante las técnicas de diagnóstico de cinta adhesiva (método de Graham) y de limpieza del área perianal con la utilización de hisopos humedecidos con solución fisiológica, hasta antes de ser infectados con huevos del parásito intestinal Syphacia obvelata, para comprobar la ausencia del parásito.

A los animales del Grupo c (inoculados con Leishmania mexicana), se inocularon a la sexta semana de edad vía intrapodal, con una cantidad de $1 \times 10^{5}$ parásitos extraídos de una lesión en un hámster. Previo a la inoculación se examinaron los ratones usando las técnicas descritas: método de Graham e hisopo húmedo, para corroborar que estos estaban libres de parásitos (Syphacia obvelata).

Los ratones alojados en los aisladores desde el destete hasta los 21 días de nacidos del Grupo D (inoculados con Syphacia obvelata más Leishmania mexicana), una vez diagnosticados libres de parásitos se infectaron con Syphacia obvelata. Luego de 21 días más, se inocularon con los parásitos de Leishmania mexicana. Este tiempo de inoculación se encuentra relacionado con los ciclos evolutivos de ambos parásitos. La obtención y la inoculación de los parásitos se llevaron a cabo usando las técnicas previamente descritas.

Para parasitar a los ratones con Syphacia obvelata, se aislaron los huevos del grupo de ratones $\mathrm{BALB} / \mathrm{c} / /$ BIOULA [13], alojados en el área experimental del Bioterio de la Universidad de Los Andes, y preparados para lograr la parasitación de estos. Se realizó semanalmente el diagnóstico de la presencia del parásito mediante el método de Graham. Una vez observados los huevos, fueron recuperados de los ratones infectados mediante la técnica del hisopo húmedo, la cual consistió en frotar la región perianal de los animales con un hisopo humedecido con solución fisiológica, luego se mezcló en un portaobjeto con una gota gruesa $(200 \mu \mathrm{L})$ de la misma solución. La muestra obtenida se observó al microscopio recorriendo toda la gota, se 
contaron los huevos, se tomó luego la gota con una jeringa de $1 \mathrm{cc}$, y se colocaron vía intragástrica a los ratones, mediante sonda gástrica. El número total de huevos administrados fue de 10 huevos por ratón.

\section{Evaluación de parámetros fisiológicos}

Luego del destete, los ratones fueron pesados y medidos semanalmente, las medidas se realizaron durante un periodo de seis semanas; para esto se utilizó una balanza marca SF-400 con una precisión de $1 \mathrm{~g}$ y una regla de medición común.

\section{Evaluación de parámetros hematológicos}

Se tomaron muestras de sangre vía retroorbital $(200 \mu \mathrm{L})$ para evaluar los parámetros hematológicos (cuenta leucocitaria, hematocrito y recuento diferencial), previo a las inoculaciones de los parásitos. A los dos meses de la posinoculación, el sangrado se realizó mediante vía intracardíaca. Para la aplicación de la técnica, se anestesiaron los animales con Enfluorano (100\%) [18], en cámara de anestesia; una vez comprobada la inconsciencia de los animales mediante la punción podal, se abrió cavidad torácica y se tomaron aproximadamente $2 \mathrm{~mL}$ de sangre, de los cuales a $1 \mathrm{~mL}$ se le colocó EDTA para realizar la hematología completa y a $1 \mathrm{~mL}$ no se le colocó EDTA para evaluar concentración de IgG mediante la técnica de inmunoensayo enzimática ELISA. El hematocrito se realizó usando una microcentrífuga (CriptSpin) durante 3 minutos a $3350 \mathrm{rpm}$. Para la determinación de la hemoglobina, se utilizó el estuche HemogloWiener Estándar, siguiendo las instrucciones del proveedor, las mediciones se realizaron a longitud de onda de $546 \mathrm{~nm}$, en un fotómetro marca Boehringer Mannheim 4010. La cuenta de glóbulos blancos se realizó mediante cámara de Neubauer y un microscopio marca Leica, visualizando la muestra a 10x; por último, la cuenta diferencial se realizó mediante coloración de frotis sanguíneos con Giemsa 10\% y contaje con la ayuda del contador diferencial Microprocessor PBE-8E.

Para el análisis inmunológico (prueba de ELISA), se obtuvo el antígeno de Syphacia obvelata. La masa de parásitos se logró de los 10 ratones BALB//C//BIOULA preparados para la obtención del antígeno. Luego de corroborada la presencia de parásitos mediante las técnicas de diagnóstico antes señaladas, los animales se anestesiaron y sacrificaron en una cámara contentiva de Enflurano; los parásitos se obtuvieron del colon y el ciego, colocándolos en una cápsula de petri, se realizó la extracción de la materia fecal de dichos órganos mediante un palillo de madera. Las muestras se colocaron en solución salina y se observaron bajo la lupa para visualizar y contar los parásitos adultos; se recolectaron uno por uno con una aguja de jeringa, en grupos de 60 a 80 parásitos adultos. Posteriormente, se colocaron en $\mathrm{PBS}$ con gentamicina $(80 \mathrm{mg} / 2 \mathrm{ml})$; se realizaron lavados a la masa de parásitos con PBs hasta obtener una masa limpia de bacterias y se guardaron en el congelador a $-40^{\circ} \mathrm{C}$ en tubos eppendorf, hasta su procesamiento.

\section{Preparación del antígeno}

Los parásitos obtenidos anteriormente fueron descongelados, luego se centrifugaron en una centrífuga refrigerada (marca Damon/IEC, modelo OPR 6000), a 2000 $\mathrm{rpm} / 5 \mathrm{~min}$. Se recolectó el sedimento contentivo de los parásitos en un solo tubo. Los parásitos se resuspendieron en $2 \mathrm{~mL}$ de PBs más $2 \mu \mathrm{L}$ de inhibidores de proteasas para sonicar posteriormente la mezcla de parásitos en un sonicador (Brason sonifer 150D), a una frecuencia de 7 hertz por 5 ciclos de 20 segundos, alternando con un minuto de descanso en baño de maría de hielo. Luego se procedió a colocar una gota sobre la lámina de un portaobjeto para observar al microscopio el rompimiento de los parásitos.

Se realizó la cuantificación de las proteínas por el método Bradford: utilizando una reacción colorimétrica visible del colorante (Coomasie G-250) leída en un espectrómetro a una longitud de onda de $595 \mathrm{~nm}$, de acuerdo con el método de Bradford. Para obtener la concentración de proteínas presente en cada una de las muestras, se dividió el valor de la absorbancia obtenido de cada muestra entre 0,06 entre los microlitros utilizados de la muestra analizada.

Luego se obtuvieron los sueros positivos y negativos, se anestesiaron y se sacrificaron los animales en una cámara con Euflurano para lograr la extracción de sangre vía intracardiaca. Un volumen de aproximadamente $1,0 \mathrm{ml}$ se colocó en tubos eppendorf sin anticoagulante EDTA para obtener suero, para el análisis inmunológico de elisa. Finalmente, el tubo se centrifugó a 1500 rpm por 5 minutos en una centrífuga marca FaNem, modelo 206 , para separar el suero $(200 \mu \mathrm{L})$. Estos se colocaron en tubos eppendorf de $1,5 \mathrm{ml}$ y se guardaron a $4{ }^{\circ} \mathrm{C}$ hasta su posterior uso. De esta manera, se obtuvieron los sueros, tanto en los animales parasitados (suero positivo) como en los animales controles (sueros negativos). 
Para la preparación del antígeno de Leishmania mexicana, se siguieron las pautas de los procedimientos expuestos, en la sección de "Preparación de antígenos". Los parásitos de Leishmania mexicana fueron donados por el doctor Juan Luis Concepción de la Facultad de Ciencias de la Universidad de Los Andes, obtenidos de un hámster con seis semanas de parasitosis.

En el ensayo inmunoenzimático, se sensibilizaron las placas de ELISA de poliestireno marca NUNCLON Surface, fondo plano, con el extracto del antígeno de Syphacia obvelata y Leishmania mexicana, a una concentración de $1 \mu \mathrm{g}$ por pozo, diluido en $100 \mu \mathrm{L}$ de buffer de unión o buffer carbonato. Posteriormente, las placas se incubaron por ocho horas a temperatura ambiente, tapadas y protegidas para evitar evaporación. Luego se realizaron tres lavados por 10 minutos cada uno, con PBs más Tween 20 (0,005\%); después de cada lavado, las placas se sacudían vigorosamente sobre un papel absorbente; luego, fueron bloqueadas con $200 \mu \mathrm{L} /$ pozo con PBS más caseína $2 \%$ y se incubaron por una hora, con el fin de bloquear los sitios libres de proteínas en el plástico. Se realizaron posteriormente tres lavados con PBs más Tween 20 (0,05\%). Los sueros se diluyeron en caseína $2 \%$ a temperatura ambiente, colocando en cada pozo las siguientes diluciones: 1:50, 1:100, 1:200, 1:400, 1:800 en volúmenes de $100 \mu \mathrm{L}$ por pozo; se incubaron por una hora a temperatura ambiente, se realizaron tres lavados por 10 minutos cada uno, con PBs más Tween $20(0,05)$ y luego se les colocó el conjugado IgG (Anti-Mouse marca Sigma) peroxidasa en una dilución 1:7500 con PBS caseína $2 \%$ en volúmenes de $100 \mu \mathrm{L}$ por pozo. Se dejó incubando por una hora, se volvieron a realizar tres lavados con PBs más Tween $20(0,005)$, se procedió a realizar el revelado de la muestra con OPD (ortofenil-dietanolamina) al 0,2\% con peróxido de hidrógeno y buffer citrato, se incubó protegido de la luz por 15 minutos y luego se paró la reacción con HCL $0,5 \mathrm{M}$ y, finalmente, se procedió a realizar la lectura de las muestras en el lector de ELISA, utilizando un filtro de lectura de $450 \mathrm{~nm}$.

Los resultados fueron analizados estadísticamente mediante ANova, aplicando el test de Dunnett para la realización de comparaciones múltiples en los diferentes grupos en estudio.

Este estudio contó con el aval del Comité de Ética del Bioterio de la Universidad de Los Andes, mediante el código CEBIOU/22-12, aprobado en mayo de 2012.

\section{Resultados y discusión}

Debido a que Falú et al. [19] demostraron que la cepa de ratones BALB/c es más susceptible a la infección por Leishmania en comparación con las cepas de ratones $\mathrm{C} 57 \mathrm{BL} / 6$; y Barreto [13] afirma que la cepa de ratones BALB/C//BIOU presenta mayor susceptibilidad a la infección producida por Syphacia obvelata con respecto a las cepas C57BL/6 y NMRI, se planteó evaluar el sinergismo en la respuesta de estos ratones ante la parasitosis combinada.

En relación con el desarrollo de la parasitosis de Syphacia obvelata, se observó (tabla 1) que tanto los machos como las hembras del Grupo в (parasitados sólo con Syphacia obvelata) y D (parasitosis combinada) desarrollaron la parasitosis. La presencia de huevos se percibió a partir de la cuarta semana, luego de la infección, encontrándose diferencias en la cantidad de huevos entre ambos grupos.

Tabla 1. Cantidad de huevos y parásitos adultos encontrados en los ratones del ensayo

\begin{tabular}{|l|c|c|c|}
\hline & & Huevo $^{*}$ & Adulto $^{* *}$ \\
\hline \multirow{2}{*}{ Grupo A } & hembras & $\mathrm{x}$ & $\mathrm{x}$ \\
\cline { 2 - 4 } & machos & $\mathrm{x}$ & $\mathrm{x}$ \\
\hline \multirow{2}{*}{ Grupo B } & hembras & 51 & 37 \\
\cline { 2 - 4 } & machos & 70 & 48 \\
\hline \multirow{2}{*}{ Grupo C } & hembras & $\mathrm{x}$ & $\mathrm{x}$ \\
\cline { 2 - 4 } & machos & $\mathrm{x}$ & $\mathrm{x}$ \\
\hline \multirow{2}{*}{ Grupo D } & hembras & 65 & 53 \\
\cline { 2 - 4 } & machos & 85 & 70 \\
\hline
\end{tabular}

${ }^{*}$ Técnica de Graham ${ }^{* *}$ Muestra fecal

Fuente: elaboración propia

La presencia tanto de huevos como del parásito adulto, en relación con el sexo de los ratones, es mayor a la cantidad en machos que en hembras, lo que está reportado por otros autores. Hagel et al. [20] presentaron la existencia de una gran susceptibilidad y daños en el hospedador por parte del parásito, dependiendo de diferentes condiciones fisiológicas, entre las que se pueden mencionar el peso, la edad, el sexo y el estado inmunitario del hospedador. Estos antecedentes fortalecen los resultados obtenidos en este estudio con respecto a una mayor susceptibilidad de los ratones machos al parásito Syphacia obvelata en los ratones $\mathrm{BALB} / \mathrm{c} / / \mathrm{BIO}$. Los ratones machos comparados con las hembras tienden a ser infectados con mayor frecuencia por el parásito, hecho que demuestra que la susceptibilidad está relacionada con el sexo del hospedador 
[21]. El presente estudio no puede demostrar la razón de esta mayor ocurrencia en hembras; es posible que ello guarde relación con la fisiología hormonal de las hembras, la cual tiene influencia en otros parámetros. Los reportes encontrados sólo mencionan este evento sin ofrecer alguna explicación, por lo que es importante hacer una investigación para determinar las variables asociadas con esta situación.

Se pudo observar, además, una mayor cantidad de huevos y parásitos en los ratones que se encontraban con la parasitosis Syphacia obvelata y Lehismania mexicana, lo que indica que la presencia de ambos parásitos en los animales llevó al aumento de la parasitosis intestinal, tanto para hembras como para los machos, siendo mayor en estos últimos.

$\mathrm{Al}$ evaluar el crecimiento en peso de los ratones $\mathrm{BALB} / \mathrm{C} / / \mathrm{BIO}$ para los tres grupos experimentales (B, C y D), en comparación con el crecimiento de los ratones control sin parasitar, no se observaron diferencias estadísticamente significativas con un $p>0,05$; sin embargo, a pesar de que la tendencia del crecimiento en el peso de los ratones, tanto machos como hembras, fue de aumento continuo durante las seis semanas de evaluación para los tres grupos experimentales, el grupo de ratones con la parasitosis combinada presentó un crecimiento inferior en los machos (figura 1).

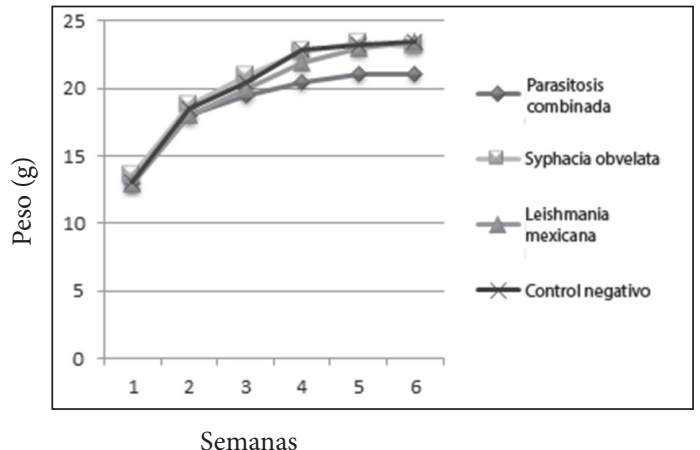

Figura 1. Curva de crecimiento de ratones BALB/c//BIO machos del grupo con parasitosis combinada y grupos controles Fuente: elaboración propia

En relación con el crecimiento en talla, este fue menor en los machos de los grupos parasitados con Syphacia obvelata y parasitosis combinadas (figura 2).

El crecimiento de los individuos generalmente se ve afectado por la presencia de parasitosis intestinales. Haber observado un menor crecimiento tanto en ganancia de peso como en ganancia de talla, en los ratones machos parasitados con Syphacia obvelata y con parasitosis combinadas con Syphacia

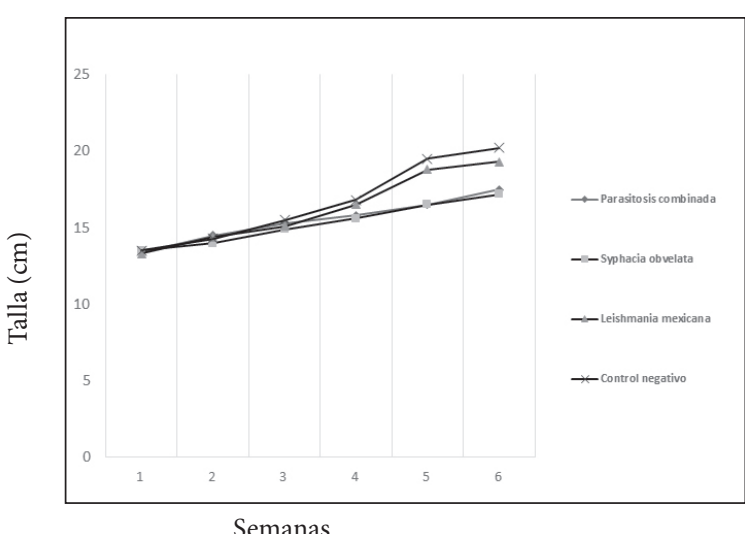

Figura 2. Curva de crecimiento de ratones BALB/c//BIO machos del grupo con parasitosis combinada y grupos controles de acuerdo con la talla

Fuente: elaboración propia

obvelata-Lehismania mexicana, confirma lo expuesto por otros autores, que dicen que la presencia de parásitos intestinales afecta el crecimiento de los animales, lo que fue corroborado en este estudio, ya que los ratones que fueron parasitados solamente con Lehismania mexicana no presentaron retrasos en el crecimiento [22].

En cuanto a la valoración de los parámetros hematológicos, la tabla 2 presenta los valores correspondientes a: hematocrito, hemoglobina, cuenta leucocitaria y recuento diferencial, encontrados en los grupos de ratones hembras y machos, controles y parasitados al inicio y al final de la experiencia, con el propósito de determinar si la presencia de parasitosis combinadas Syphacia obvelata y Leishmania mexicana alteraba los parámetros hematológicos.

En el análisis estadístico ANOva aplicado a los datos de los cuatro grupos (A, B, C y D) en estudio, se encontraron diferencias estadísticamente significativas $(p<0,05)$. Aplicando la prueba de Tukey para determinar cuál era la media que conllevaba este resultado, se encontró que, para los valores de hematocrito, hubo coincidencia entre los grupos A - B y C - D, por lo que se podía considerar que no existe sinergismo con respecto al parámetro hematocrito. En relación con la hemoglobina, se observó igualmente coincidencia entre las medias de los grupos A - С у в - D, al igual que se puede considerar que no existe sinergismo. Sin embargo, al valorar mediante la prueba de Tukey los parámetros relacionados con la cuenta leucocitaria y a la diferencial, se encontró que el grupo $\mathrm{D}$ fue el que presentó diferencias en la media, considerándose que existe un sinergismo con respecto a estos parámetros. 
Tabla 2. Promedios y desviación estándar de los valores hematológicos de los grupos en experimentación

\begin{tabular}{|c|c|c|c|c|c|}
\hline & & Grupo A & Grupo B & Grupo C & Grupo D \\
\hline \multirow{2}{*}{ Hematocrito (\%) } & Machos & $35 \pm 3,2$ & $31 \pm 3,4$ & $27 \pm 3,4$ & $27 \pm 3,5$ \\
\hline & Hembras & $28 \pm 5,6$ & $28 \pm 11,1$ & $23 \pm 5,8$ & $21 \pm 4,6$ \\
\hline \multirow{2}{*}{$\begin{array}{l}\text { Hemoglobina } \\
(\mathrm{g} / \mathrm{dL})\end{array}$} & Machos & $19 \pm 2,3$ & $12,5 \pm 2,5$ & $15,4 \pm 1,2$ & $12 \pm 4,9$ \\
\hline & Hembras & $17 \pm 1,7$ & $13,9 \pm 6,7$ & $15 \pm 3,7$ & $13 \pm 2,7$ \\
\hline \multirow{2}{*}{$\begin{array}{l}\text { Cuenta leucocitaria } \\
/ \mathrm{mm}^{3}\end{array}$} & Machos & $4150 \pm 456,3$ & $4900 \pm 345,9$ & $6200 \pm 367,9$ & $8300 \pm 378,9$ \\
\hline & Hembras & $3600 \pm 23,4$ & $4200 \pm 137,9$ & $5600 \pm 278,2$ & $6500 \pm 287,1$ \\
\hline \multirow{2}{*}{$\begin{array}{l}\text { Linfocitos } \\
(\%)\end{array}$} & Machos & $97 \pm 5,8$ & $90 \pm 2,5$ & $85 \pm 3,6$ & $80 \pm 3,7$ \\
\hline & Hembras & $93 \pm 0,8$ & $83 \pm 6,9$ & $80 \pm 3,9$ & $78 \pm 2,8$ \\
\hline \multirow{2}{*}{$\begin{array}{l}\text { Neutrófilos } \\
(\%)\end{array}$} & Machos & $11 \pm 7,1$ & $10 \pm 1,3$ & $17 \pm 2,7$ & $10 \pm 1,9$ \\
\hline & Hembras & $15 \pm 3,2$ & $12 \pm 1,9$ & $10 \pm 1,5$ & $18 \pm 3,6$ \\
\hline \multirow{2}{*}{$\begin{array}{l}\text { Eosinófilos } \\
(\%)\end{array}$} & Machos & $0 \pm 0,0$ & $5 \pm 0,9$ & $2 \pm 0,9$ & $6 \pm 0,9$ \\
\hline & Hembras & $0 \pm 0,0$ & $8 \pm 0,6$ & $3 \pm 0,5$ & $7 \pm 0,5$ \\
\hline
\end{tabular}

Fuente: elaboración propia

Tanto los valores bajos de hematocrito como de hemoglobina puede ser, en forma general, indicadores de anemias. Esta condición se presenta en el grupo D, y de acuerdo con la prueba de comparación de medias en el caso del hematocrito, la similitud de medias del grupo C y el D hace suponer que la influencia de la parasitosis con Leishmania mexicana afecta más a este parámetro. En cuanto al parámetro hemoglobina, la relación de medias fue entre los grupos D y в, suponiendo que este parámetro es afectado mayormente por la Syphacia obvelata. En ambos parámetros, la tendencia fue la de disminuir los valores.

Estos resultados, de acuerdo con diferentes reportes, se deben a la presencia del parásito intestinal, ya que se ha comprobado que la adhesión de los parásitos helmintos en las vellosidades intestinales puede causar alteración en los valores hematológicos del hospedador provocando la disminución de los niveles de hemoglobina, ocasionada ya sea por acción mecánica, enzimática o química del parásito, lo que causa daño intestinal. Su gravedad está relacionada directamente con la carga parasitaria del hospedador debido a que existe un aumento del daño en el intestino por la gran cantidad de parásitos [23].

Otra posible causa de la disminución de los valores de hemoglobina en ratones parasitados con la Syphacia obvelata puede ser que, a nivel del tracto digestivo grueso, se sintetiza un factor intrínseco que permite la absorción de la vitamina B12; por tanto, si esta síntesis se ve afectada, no producirá el factor de absorción, lo que a su vez disminuiría la absorción de vitamina B12 para la producción de eritrocitos en la médula y llevará a la disminución de hemoglobina causando anemia [24].

Con respecto a los valores promedios del recuento leucocitario de los ratones machos y hembras del Grupo control en comparación con los grupos parasitados B, C y D, al aplicar el análisis estadístico ANOva se encontró que existen discrepancias estadísticamente significativas en los diferentes grupos. Al realizar la prueba de Tukey, las medias que determinaron las diferencias significativas fueron las del grupo D. En el caso de la cuenta leucocitaria, esta fue mayor; en el caso de los neutrófilos, fue menor, y en el caso de los eosinófilos, se observó la condición de eosinofilia en relación con el Grupo control como con los grupos parasitados (A у в). Estos resultados son conducentes para hablar de un sinergismo de la respuesta de los animales al tener una parasitosis combinada: Leishmania mexicana y Syphacia obvelata.

Los leucocitos forman parte de las células efectoras del sistema inmunológico; con capacidad migratoria, utilizan la sangre como vehículo para acceder a las diferentes partes del cuerpo. Los leucocitos son los encargados de destruir los agentes de infección y las células infectadas, secretando sustancias protectoras como los anticuerpos y combatiendo las infecciones [25].

$\mathrm{Al}$ observar la cuenta leucocitaria en los grupos de ratones machos y hembras, BALB/c//BIOULA, se pudo contrastar lo expuesto, en donde el organismo al sufrir una invasión por agentes extraños aumenta considerablemente las células efectoras del sistema 
inmunológico. Los eosinófilos son células presentes en la sangre que se encargan de actuar frente a sustancias extrañas (bacterias, parásitos, entre otras) que penetran el organismo, ocasionando inflamación; su número aumenta debido a la presencia del parásito. En las infecciones parasitarias producidas por helmintos, la activación de neutrófilos y eosinófilos tiene un papel efector importante. Por tanto, el aumento de los eosinófilos en los ratones parasitados, tanto machos como hembras de los diferentes grupos de ratones $\mathrm{BALB} / \mathrm{c} / /$ BIOULA parasitados, se debe a la presencia del parasito intestinal Syphacia obvelata y del protozoario Leishmania mexicana. Los helmintos desencadenan una serie de respuestas inmunológicas en el hospedador producto de su invasión y multiplicación, como la secreción de citocinas por las células Th2 con una producción elevada de IL-4, IL-5 y IL-13 [26]. Las células efectoras como los macrófagos, neutrófilos, eosinófilos y mastocitos pueden atacar a los parásitos; por tanto, el nivel de estas células aumenta en presencia de infecciones parasitarias [27]. Los ratones $\mathrm{BALB} / \mathrm{c}$ infectados con Leishmania presentan una respuesta inmunológica de tipo Th2, que es estimulada por la producción de IL-4 y IL-5. Estas a su vez activan las células neutrófilas en mayor proporción y los eosinófilos en menor cantidad; ambas contribuyen en la respuesta inflamatoria, respuesta innata que desencadena el sistema inmunológico para combatir la infección parasitaria [28]. Esto explica los resultados encontrados en los diferentes grupos de ratones BALB/c//BIOULA, en los que los niveles de neutrófilos y eosinófilos aumentaron considerablemente en los ratones parasitados en relación con el grupo control sin infección, hechos que demuestran que las infecciones parasitarias ocasionadas por Syphacia obvelata y Leishmania mexicana alteran los valores hematológicos de cada animal.

\section{Análisis inmunológico (prueba ELISA)}

Al ser el parasitismo un fenómeno biológico interdependiente y dinámico, la infección puede provocar escasas manifestaciones; sin embargo, el estado inmunitario del huésped siempre da una respuesta, debido a la misma dinámica de los eventos que ocurren en el establecimiento de la infección. La acción inmunológica fue otro de los parámetros valorados en el estudio para observar si existía un sinergismo entre la infección por el parásito Syphacia obvelata (parásito intestinal común en los bioterios convencionaes) y la Lehismania mexicana (parásito intracelular comúnmente usado en estudios de parasitosis experimental en el modelo ratón consanguíneo BALB/c).

En el desarrollo de esta parte experimental, en la estandarización de la técnica de ELISA, se usaron muestras de suero. El suero del grupo control negativo presentó valores entre 0,01 y 0,03 de densidad óptica, y para las del grupo control positivo, se encontraron valores superiores a 0,1 de densidad óptica. Los resultados de sueros negativos y positivos se correlacionaron con la ausencia y presencia de los parásitos involucrados en el estudio, validados mediante las pruebas de diagnóstico comunes: cinta adhesiva (técnica de Graham) y presencia de parásitos en muestras frescas para Syphacia obvelata, mediante frotis sanguíneos para la presencia de la Lehismania mexicana y la ausencia por la no presencia de los parásitos al desarrollar estas técnicas.

La tabla 3 presenta los valores de la concentración de anticuerpos IgG, para los sueros provenientes de ratones del Grupo control (Grupo A) sin parasitar, y de los Grupos в (parasitados con Syphacia obvelata), Grupo C (parasitado con Lehismania mexicana) y el Grupo D (parasitosis combinadas: Syphacia obvelata y Lehismania mexicana).

Tabla 3. Medidas de concentración (unidades) de anticuerpos IgG, encontradas para los grupos involucrados en la experiencia

\begin{tabular}{|l|l|l|l|l|l|}
\hline \multicolumn{2}{|l|}{$\begin{array}{l}\text { Medidas de absorbencia a } \\
450 \mathrm{~nm}\end{array}$} & $\begin{array}{l}\text { Grupo A } \\
\text { (sin parásitos) }\end{array}$ & $\begin{array}{l}\text { Grupo B } \\
\text { (Syphacia obvelata) })\end{array}$ & $\begin{array}{l}\text { Grupo C } \\
\text { (Lehismania } \\
\text { mexicana) }\end{array}$ & $\begin{array}{l}\text { Grupo D (parasitosis } \\
\text { combinada) }\end{array}$ \\
\hline $\begin{array}{l}\text { Placas sensibilizadas con } \\
\text { Syphacia obvelata }\end{array}$ & Machos & 0,01 & 0,13 & 0,01 & 0,18 \\
\cline { 2 - 7 } & Hembras & 0,03 & 0,13 & 0,01 & 0,18 \\
\hline $\begin{array}{l}\text { Placas sensiblizadas con } \\
\text { Lehismania mexicana }\end{array}$ & Machos & 0,03 & 0,03 & 0,13 & 0,17 \\
\cline { 2 - 7 } & Hembras & 0,03 & 0,03 & 0,13 & 0,17 \\
\hline
\end{tabular}

Fuente: elaboración propia 
Se puede observar en la tabla que los valores de concentración de anticuerpos contra el parásito Syphacia obvelata en las placas sensibilizadas con este parásito presentaron valores de $0,13 \mathrm{~nm}$ tanto para machos como para hembras, indicando presencia de anticuerpos contra el parásito. Para los sueros de los ratones parasitados con Leishmania mexicana, tanto para machos como para hembras, se encontraron concentraciones de 0,03 indicando la no presencia de anticuerpos contra la Syphacia obvelata.

En el caso de las placas sensibilizadas con el parásito Lehismania mexicana, los ratones del grupo control в (inoculado con Syphacia obvelata) no mostraron la presencia de anticuerpos contra este, y el suero de los ratones parasitados con Lehismania mexicana presentó la presencia de anticuerpos.

En relación con el grupo D (ambas parasitosis), en la placa sensibilizada con Syphacia obvelata y en la sensibilizada con Lehismania, los ratones de este grupo mostraron las mayores concentraciones de $\operatorname{IgG}(0,18$ y 0,17 , respectivamente), tanto para hembras como para machos. Estos resultados permitieron observar la presencia de sinergismo. Al aplicar el análisis estadístico con $p<0,05$, se demuestra que hay diferencias estadísticamente significativas entre los dos grupos en estudio, con base a los niveles de IgG.

En estudios anteriores: Gudiño et al. [12] determinaron que era posible diagnosticar la presencia de Shyphacia obvelata mediantela técnica de ELISA; Barreto [13] determinó que la cepa consanguínea BALB/c//BIO era más susceptible a la infección de Syphacia obvelata que la cepa consanguínea C57BL/6//BIO y BIO:NMRI.

La respuesta inmunológica del ratón BALB/c está relacionada con la activación de los linfocitos Th2, con producción de anticuerpos e interleucinas como la IL-4 (respuesta Th2); podría aumentar la expresión del HLA-II, la producción de IgG e IgE y el crecimiento de células $\mathrm{T}$ [29], determinada igualmente en estudios con Lehismania mexicana de Castes y Tapia [30-32] en esta cepa de ratones.

En este estudio, al analizar los valores de la concentración de IgG que se obtuvieron mediante la técnica de ELISA, se logró demostrar la existencia de sinergismo en los ratones BALB/c//BIOULA en la parasitosis combinada: Syphacia obvelata y Leishmania mexicana, y se encontró mayor valor de densidad óptica en los sueros del Grupo control в (inoculados con Syphacia obvelata), Grupo control c (inoculados con Leishmania mexicana) y Grupo experimental (ambas parasitosis). Se descubrió una mayor concentración de anticuerpos frente a los parásitos en esta condición. $\mathrm{Al}$ analizar estadísticamente los distintos grupos, se encontraron diferencias significativas con $p<0,05$ entre la cantidad de anticuerpos contra el parásito en cada uno de los grupos de ratones $\mathrm{BALB} / \mathrm{c} /$ /BIOULA parasitados.

\section{Conclusiones}

Existe una sinergia en la respuesta de los ratones $\mathrm{BALB} / \mathrm{c} /$ BIOULA cuando tienen una parasitosis combinada entre Syphacia obvelata y Leishmania mexicana, para los parámetros de la cuenta leucocitaria, la cuenta diferencial y en la respuesta inmunológica relacionada con la concentración de IgG, ya que los valores encontrados son mayores que al momento de encontrarse parasitados con Syphacia obvelata o Leishmania mexicana. Se puede observar igualmente el sinergismo en la respuesta de los ratones que tienen parasitosis combinadas cuando se valora el crecimiento de los animales, ya que es menor que al momento en que los ratones se encuentran parasitados con un solo parásito, y en la mayor cantidad de huevos adultos de la Syphacia obvelata que se observan en estos ratones. Los resultados obtenidos permiten alertar a los investigadores usuarios de los animales sobre la importancia de trabajar con ratones que se encuentren parasitados con Syphacia obvelata, ya que desconocer este acontecimiento puede llevarles a una conclusión basada en premisas alteradas. Los animales que se utilicen para estudios sobre Leishmaniasis deben encontrarse libres de oxiuros.

\section{Agradecimientos}

Este trabajo fue financiado parcialmente por el Centro de Desarrollo Científico, Humanístico, Tecnológico y de las Artes de la Universidad de Los Andes, Mérida, Venezuela, mediante los proyectos: C-1789-12-03-F y SE-C-02-14-03

\section{Referencias}

[1 Northrop-Clewes C, Rousham E, Mascie-Taylor C, Lunn PG. Anthelmintic Treatment of Rural 
Bangladeshi Children: Effect on Host Physiology. Am J Clin Nutr. 2001;73(1):53-60.

[2] Babu S, Bhat S, Kumar NP, Kumaran P, Gopi PG, Kolappan C, Kumaraswami V, Nutman TB. Human Type 1 and 17 Responses in Latent Tuberculosis are Modulated by Coincident Filarial Infection through Cytotoxic T Lymphocyte Antigen-4 and Programmed Death-1. J. Infe. Dis. 2009;(2):288-98.

[3] Pereira E, Silva Y, Secor E, Orsine A, Katz N, Rabello A. Circulating Antigens Levels in Different Clinical Forms of the Schistosoma mansoni Infection. Mem. Inst. Oswaldo Cruz. 1999;94(1):83-6.

[4] Stanley A. Balancing Immunity and Pathology in Visceral Leishmaniasis. Immunity Cell Biology. 2006;85(2):138-47.

[5] lexander J, Kaye P. Immunorregulatory Pathways in Murine Leishmaniasis: Different Regulatory Control during Leishmania mexicana mexicana and Leishmania major Infections. Clin Exp Immunol. 1985;61(3):674-82.

[6] Pérez G, Redondo G, Sacerio M, González O. Prevalencia de parasitismo intestinal en escolares de 6-11 años. Medisan. 2012;16(4):551.

[7] Preston P, Dumonde D. Experimental Cutaneous Leishmaniasis v: Protective Immunity in Subclinical and Selfhealing Infection in the Mouse. Clinical and Experimental Immunology. 1976;23(1):126-138.

[8] Cheng-Ng R, Castellano J, Díaz O, Villalobos P. Prevalencia de Giardiasis en hogares de cuidado diario en el municipio San Francisco, estado Zulia, Venezuela. Invest Clin. 2002;43(2):4-8.

[9] Anderson R. Nematode Parasites of Vertebrates. J immunol 2006;245(1):2321-28.

[10] Artis D. New Weapons in the War on Worms: Identification of Putative Mechanisms of Immune-Mediated Expulsion of Gastrointestinal Nematodes. Int J Parasitol. 2006;36(6):723-33.

[11] Noble E. The Biology of Animal Parasites. Parasitology. Philadelphia: Lea \& Febiger; 1989.

[12] Gudiño M, De Jesús R, Osorio A, Urdaneta H, Alfonso N, Mora N, O'Callaghan J. Implementación de la técnica inmunoenzayoenzimática ELISA como método de diagnóstico para la detención de Syphacia obvelata en ratones producidos para uso experimental. Parasitol Latinoam. 2009; 61(1):86-91.

[13] Barreto A. Determinación de la susceptibilidad a la infección de Syphacia obvelata en ratones consanguíneos y no consanguíneos [tesis de grado]. Universidad de Los Andes; 2012.

[14] Cunningham, J. Fisiología veterinaria. Madrid: Editorial Elsevier; 2003.

[15] Benavides F, Guénet JL. Manual de Genética de roedores de laboratorio: principios básicos y aplicaciones. Alcalá de Henares: Sociedad Española para las Ciencias del Animal de Laboratorio; 2003.

[16] Espinoza Y, Huapaya P, Suárez R, Chávez V, Sevilla C, Dávila E, Huiza A, Naquira C, Alva P. Estandarización de la técnica de ELISA para el diagnóstico de toxocariasis humana. An Fac Med. 2003;64(1):7-12.

[17] Contreras M, Acevedo E, Aguilera S, Sandoval L, Salinas P. Estandarización de ELISA IgM e IgA para el inmunodiagnóstico de triquinosis humana. Journal Parasitol. 2000;54(3-4):104-9.

[18] Martínez M, Buzaleh A, Batlle A. Efecto de los anestésicos Enflurano e Isoflurano en ratones con niveles inducidos y deprimidos de citocromo P450: estudios sobre el sistema enzimático metabolizante de drogas. Acta Bioquim Clin Latinoam. 2005;39(1):37-42.

[19] Falú M, García MF, Parodi CM, Molina de Raspi E, Cimino R, et al. Susceptibilidad de distintas cepas de ratones a la infección por Leishmania amazonenses. Dermatol. Argent. 2009;15(5):334-9.

[20] Hagel I, Salgado A, Rodríguez D, Ortiz D, Hurtado M, Puccio F, et al. Factores que influyen en la prevalencia e intensidad de las parasitosis intestinales en Venezuela. Gac Méd Caracas. 2001;109(1):82-90.

[21] Robert T, Russell D. Harlan Health Reporting [internet]. 2006 [citado 2014 julio]. Disponible en http://Harlan.com/health/report/

[22] Clarke C, Perdue K. Detection and Clearance of Syphacia obvelata Infection in Swiss Webster and Athymic Nude Mice. Contemp Top Lab Anim Sci. 2004;43(4):9-13.

[23] Carrada-Bravo T. Uncinariasis: ciclo vital, cuadros clínicos, patofisiología y modelos de animales. Rev Mex Patol Clin. 2007;54(4):187-99. 
[24] Gonenc B, Sarimehmetoglu H, Ica A, Kozan E. Efficacy of Selamectin against Mites (Myobia musculi, Mycoptes musculinus and Radfordia ensifera) and Nematodes (Aspiculuris tetraptera y Syphacia obvelata) in Mice. Lab Anim. 2006;40(2):210-3.

[25] Lodish H, Berk A, Zipurski L, Matsudaira O, Baltimore D, Darnell J. Biología celular y molecular. Buenos Aires: Editorial Médica Panamericana; 2002.

[26] Dunne, D. A Worm's Eye View of the Immune System: Consequence for Evolution of Human Autoimmune Disease. Nat Rev Immunol. 2005;5(5): 420-26.

[27] Sato Y, Kean H, Nonaska N, Oku Y, Kamiya M. Antibody Production in Syphacia obvelata Infeccted Mice. J Parasitol. 1995;81(1):559-62.
[28] Bogdan C, Röllinghoff M. The Immune Response to Leishmania: Mechanisms of Parasite Control and Evasion. Int J Parasitol. 1998;28(1):121-34.

[29] Silvana A, Godoy A, Einisman H, Garcia D, Harris P. Regulación de la respuesta inmune frente a la infección por Helicobacter pylori. Revista Chilena de Pediatría. 2002;73(2):108-15.

[30] Castes M, Tapia F. Inmunopatolia de la Lehismaniasis tegumentaria americana. Acta Científica Venezolana. 1998;49(1):42-56.

[31] Zerpa O, Convit J. Lehismania cutánea difusa en Venezuela. Gazeta Médica de Bahía. 2009;79(Supl.3):30-4.

[32] Caraballo L, Zakzuk J. Consideraciones sobre la evolución de la respuesta immunitaria Th2 y sus posibles relaciones con parasitosis. Biomédica. 2012;32(1):145-57. 